\title{
Web-Based COOP Training System to Enhance the Quality, Accuracy and Usability Access
}

\author{
Amr Jadi \\ Department of Computer Science and Engineering \\ College of Computer Science and Engineering, University \\ of Hail, Hail, Saudi Arabia
}

\begin{abstract}
In this paper, a web based COOP training system is demonstrated to ensure usable process of task interactions between various participants. In the existing method various issues related with the paper work, communication gap, etc. raised serious issues between the colleges and industries while implementing the COOP training programs. The primary data was collected by conducting interviews with the supervisors and also by taking the opinion of students to improve the proposed COOP system. The proposed system is capable of reducing the complexity of operations to a greater extent by avoiding overlapping of the information, reducing the communication gap and by increasing the accuracy of the information. The outcomes of the proposed system proved to be very fruitful in terms of results obtained from the point of view of all the participants in the COOP system. The performance, accuracy, quality and assessment of the student reports found to be improved to deliver excellent results.
\end{abstract}

Keywords-COOP training; web applications; integration; quality; accuracy

\section{INTRODUCTION}

The training system in Kingdom of Saudi Arabia (KSA) is considering various modifications to meet the demands of current trends and to enhance the quality of education system under various trending circumstances. The introduction of Cooperating (COOP) training for the graduates made mandatory to improve the skill set as required by any industrial unit. In this COOP training, the student n3eed to attend relevant industry to learn and work as a temporary employee and then they need to perform the task as per the guidelines of the company.

There are various objectives of the COOP training system includes:

- to develop different types of practical skills those are needed by real-time applications,

- to implement various applications from the knowledge acquired from different domains,

- to evaluate the attitude and working environment,

- to interpret the learning outcomes of the university studies with the applications of real-time activities,

- to prepare a comprehensive report on the overall training learning outcomes, and

\author{
Eesa A. Alsolami \\ Department of Information Technology \\ College of Computing and Information Technology \\ University of Jedddah, Saudi Arabia
}

- to present the working skills obtained along with the experience obtained out of the learned skills.

In the Section II, a detailed activity of COOP training system is explained for an easy understanding of each participant and their roles.

\section{CONCEPT OF COOP TRAINING SYSTEM}

To obtain the above objectives of the COOP training system various participants with defined responsibilities are being allotted to perform by various educational managements and industry authorities as shown in Fig. 1. The list of participants includes a) Employer, b) Site Supervisor, c) COOP Training Coordinator, d) Faculty Advisor, and e) COOP Examining Committee.

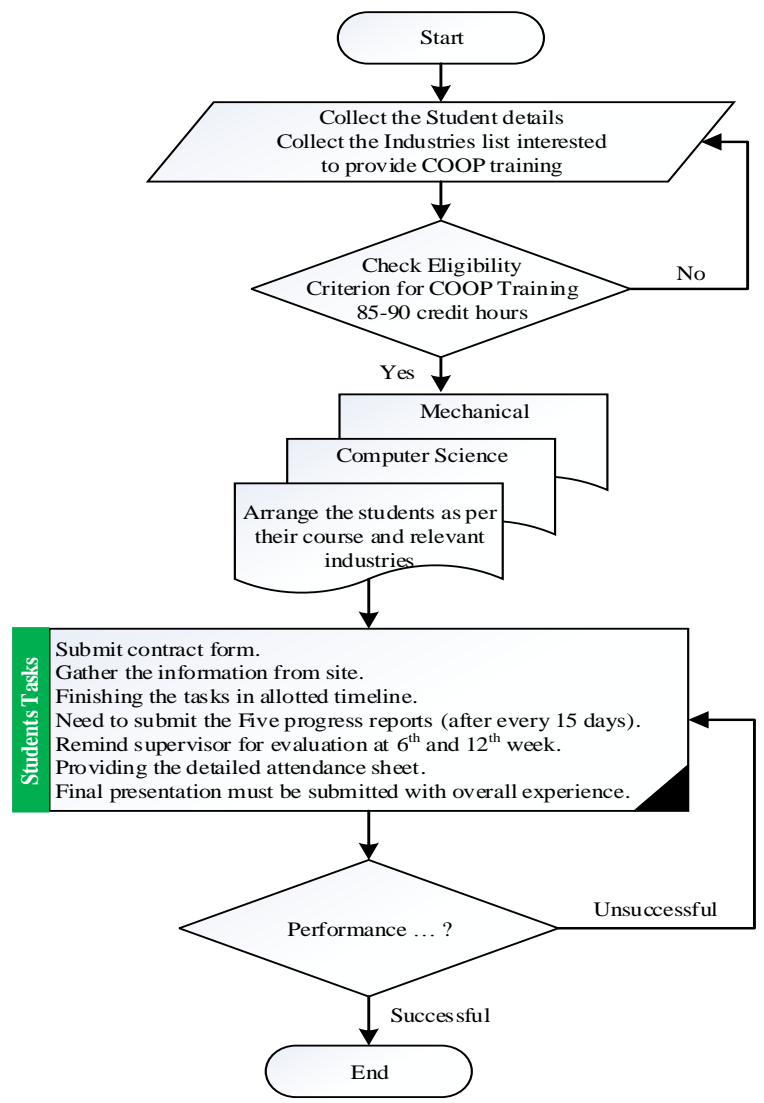

Fig. 1. Functional flow diagram of the COOP training system. 


\section{A. Employer}

An employer is considered to be the key player in the whole COOP training system for promoting a successful training program for the students and thereby achieve common goals of an educational institution with following aspects [1].

1) Need to provide a training / task plan for a period of 12 weeks related with the student's area of study and it has to be approved by faculty advisor.

2) To accomplish the given task a site supervisor must be assigned for making the training program more meaningful and effective.

3) Needs to monitor and report any kind of irregularities by the students in the organization with the faculty advisor so as to implement the corrective actions are taken by the students advisor and the same will be reflected in the evaluation reports.

4) A proper communication and visiting hours must be allotted for the faculty advisors.

\section{B. Site Supervisor}

A site supervisor is a professional within the area of student area of study. Plays a critical role in developing the student in the assigned project area with the given tasks and in given time durations [1].

1) Ensures a proper direction for the students in the training area for the guidelines given by company and faculty advisor.

2) Communicates the performance of student with employer and faculty advisor for corrective actions.

3) Encourages the students for preparing technical reports and to conduct oral presentations.

4) Ensures quality and accuracy for the bi-weekly reports submitted by students.

5) Helps the students to prepare mid-point (6 weeks) and final (12 weeks) evaluation reports for the COOP training period.

\section{COOP Training Coordinator}

The COOP training coordinator plays an important role in providing various bridging activities between the educational institutions and industries with relevant subject areas for different student courses [1].

1) A coordinator will contact the companies to ensure the slots are available and booked for the COOP training sessions.

2) Assigns a meaningful coordination between the students and qualified experts from industry to meet the requirements of student courses and given tasks.

3) Necessary information will be gathered and shared with students for the employment in COOP training program.

4) Communication between the students and employers will be established through proper channel to ensure timely training sessions.

5) Coordinates the evaluation forms from employers and faculty advisors.
6) Collects, compiles and submits the final grades to concerned departments.

\section{Faculty Advisor}

The faculty advisor is selected by the COOP training committee based on the subject, assignment, and the experience in the subject area [1]. The role of a faculty advisor includes.

1) To ensure proper work assignments, assessment of task plans, progress and student activities, and finally taking appropriate decisions during the course time by providing feedback to students, management and industry with the progress report results.

2) Providing the guidelines for the students with proper report submitting guidance and professional formats.

3) Involving positively and evaluating student presentations in necessary.

\section{E. COOP Examining Committee}

Two or more faculty members are assigned to examining the student performance during COOP training. This committee evaluates the performance reports, presentations and feedback from the COOP coordinators [1].

In Section III, a detailed study on the existing method of implementing the COOP training system is discussed with considerable factors. Based on the discussions a suitable and comparably faster approach to realize an accurate COOP training system is proposed in this work.

\section{SYSTEM CONCEPT}

Functioning of COOP training system representation is shown in Fig. 2 with existing method and in Fig. 3 with proposed web based method. Both the methods are explained in detail in the following discussions.

\section{A. Existing COOP Training System}

In the existing COOP training system (see Fig. 2) the interaction between each participant can be independent and might lead to a worst scenario at times due to lack of proper communication or missing information. The data sharing between each participant right from faculty advisor to site supervisor and to students may get into a murkier situation whereby both institution and organization providing the facilities for COOP training may get into serious conflicts.

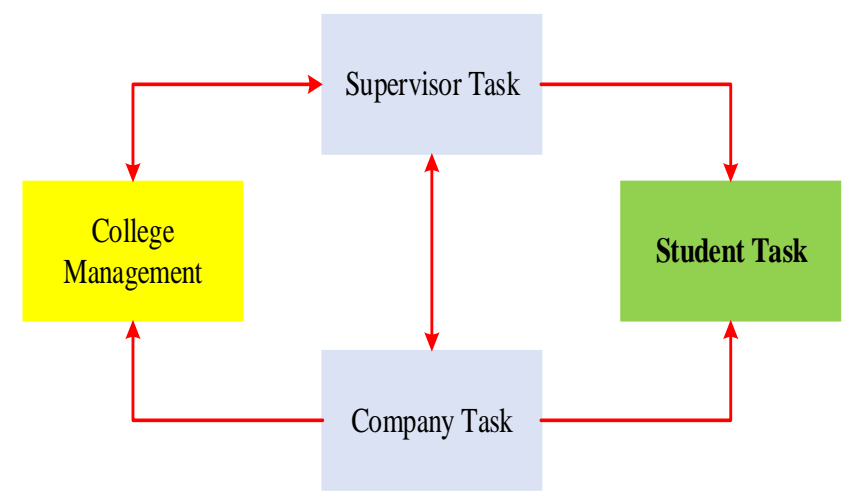

Fig. 2. Representation of existing COOP training system. 
The managements of universities and companies get to know unrealistic information from the ground truths. The probability of biased evaluations is always a threat and impact (i.e. the quality of training or improving the student skills for real time problems will affect seriously) is reasonably known to all the participants involved in education system and industry. Now such issues must be dealt with care and need to ensure an efficient COOP training system.

\section{B. Proposed Web-based COOP Training System}

The proposed COOP system as shown in Fig. 3 consists of a web-based internal communication system which collects all the information from different participants and allows them to submit their activity information using the system. A detailed tasks and communication established between all the participants are shown in the sequence diagram (see Fig. 4) using the proposed web based COOP training system.

The integration of each activity in the proposed method will be stored in a database with appropriate measure to secure the information of all the participants in the COOP system. The list of companies selected by the supervisors will be uploaded into the website by the supervisor using his login ID and the same information can be communicated to the companies, college management and students if it is related with them. Similarly, the training coordinator from the company can provide the details of the training procedures, objectives, requirements, etc. and upload them on the web portal to communicate with the relevant participants. In the process a student also can upload the project reports, etc.

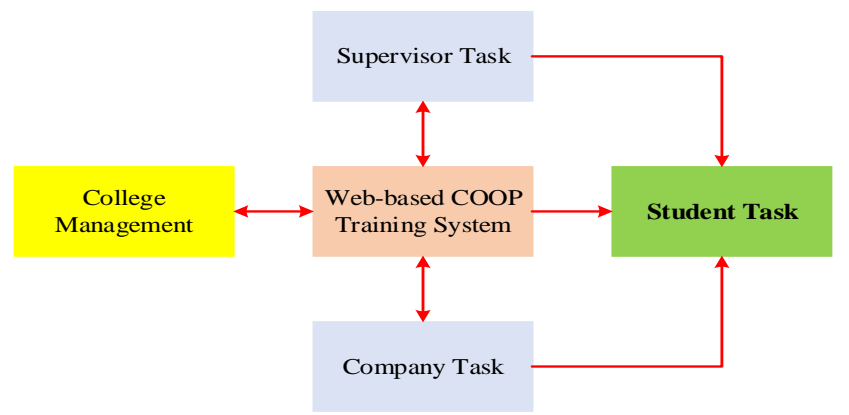

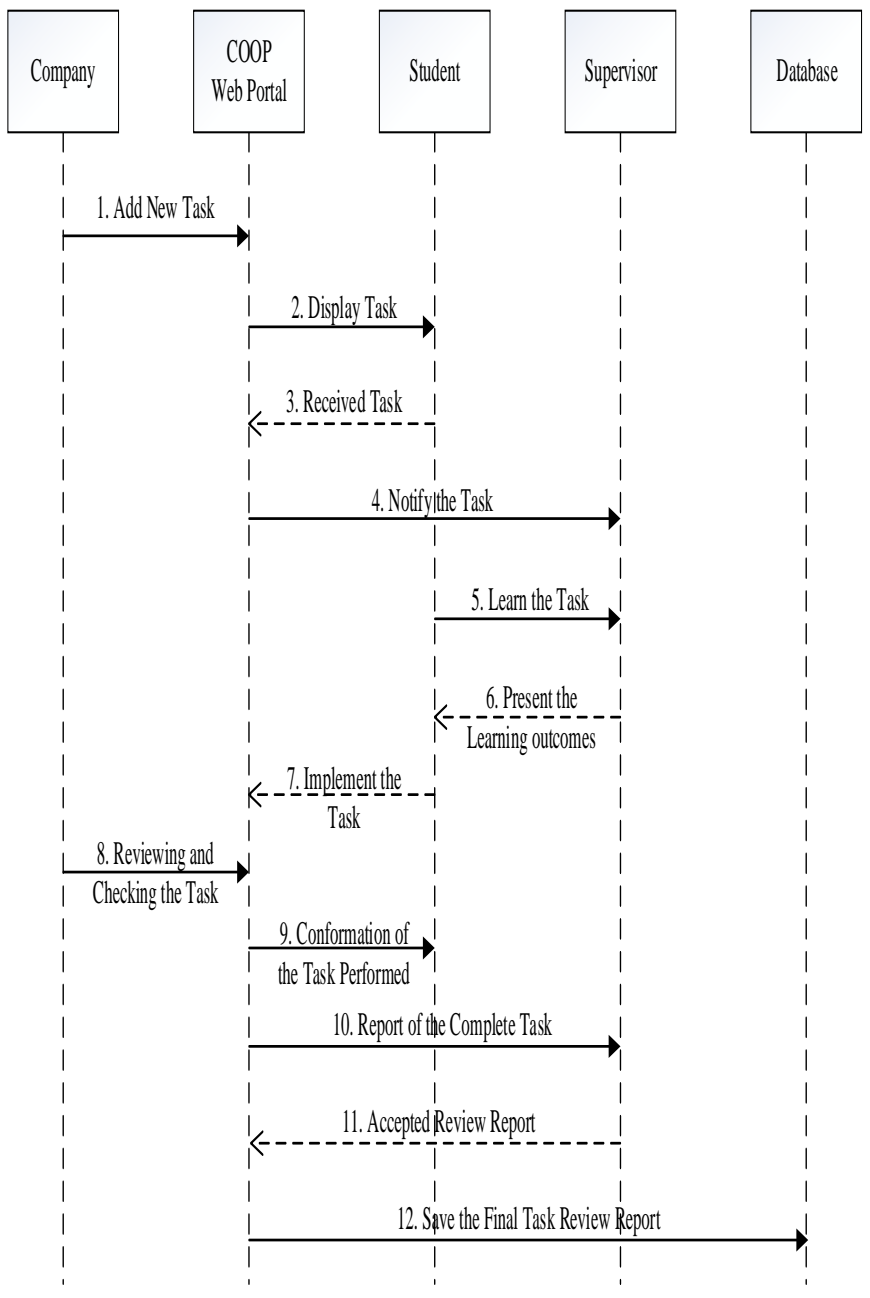

Fig. 4. Sequence diagram representing the tasks and communication between different participants using web based COOP Training System.

Similarly, the role of each participant in the web based COOP training system is summarized in Table I.

Fig. 3. Proposed web based COOP training system.

TABLE I. THE ROLE OF EACH INDIVIDUAL IN COOP TRAINING SySTEM

COOP Training System

\begin{tabular}{|c|c|c|}
\hline Supervisor Task & Company Task & Student Task \\
\hline $\begin{array}{l}\text { - Login to system } \\
\text { - Can add new student } \\
\text { - Can edit task or revive information from } \\
\text { the company } \\
\text { - Can search the status of student } \\
\text { performance, attendance and Discipline. } \\
\text { - Can update the information (deleting old } \\
\text { student information, entering new data, } \\
\text { etc.) }\end{array}$ & $\begin{array}{l}\text { - Login to system } \\
\text { - Can add new student or task } \\
\text { - Can edit task or revive task information } \\
\text { - Can search the status of student } \\
\text { performance, attendance and Discipline. } \\
\text { - Can update the information (deleting old } \\
\text { student information, entering new data, } \\
\text { etc.) }\end{array}$ & $\begin{array}{l}\text { - Login to system } \\
\text { - Can add his project reports } \\
\text { - Can edit and view his own reports } \\
\text { - Can search the schedules, tasks, grades, } \\
\text { etc. } \\
\text { - Can communicate with the site } \\
\text { supervisors, employers, training } \\
\text { coordinators, examining committee, etc. }\end{array}$ \\
\hline
\end{tabular}


The proposed COOP training system based on Web 2.0 [2] is proved to be a well suited tool for teaching and training using social networking as a tool. Various perceptions, attitude and acceptance for the Web 2.0 in the COOP training system were discussed in their work to improve the learning quality of the students. At the same time the information sharing, learning experiences, communicating information, assessment requirements and moral support will increase using web based system. It helps both industries and college staff members to identify, understand and communicate different tasks and roles of jobs to do under the COOP system. The proposed web based system helps them to develop and communicate their decisions towards other participants in the COOP system very effectively so that the quality of training and education can be evaluated easily. Most importantly, the management of both industry and university can access the proceedings of training program without any kind of delays as seen in the case of traditional methods, where the information will be in distributed form with different people and hence it used to take a lot of time as discussed earlier. Aleisa and Alabdulahfez reported spectacular growth of the student's performance and employee satisfaction in their research findings [3]. However, the same report also highlighted the challenges in terms of securing the placements in various sectors along with financial resources and personal information. The challenges were highlighted in the work of Weber using web technologies include the computer literacy, lack of Arabic language learning objects, interoperability issues, cross-platform issues, etc. in the Gulf countries [4]. The important factor for a rejection for the COOP training system by most of the people found to be due to lack of awareness of internet usage and various applications related to internet and computer based training activities in various Gulf countries [4]. Such a situation of the students completing the graduation with no computer and internet skills lead to a worst situation for most of the private employees. In a recent report, world economic forum criticized the inadequate educated workforce as a major problem in Qatar to do any kind of business. Hence, having COOP training programs will encourage both the industries and colleges to obtain encouraging results out of present demand for the qualified workforce [4]. The usage of web based learning has seen a grater improvement according to Gulf Cooperation Council (GCC) as shown in Fig. 5.

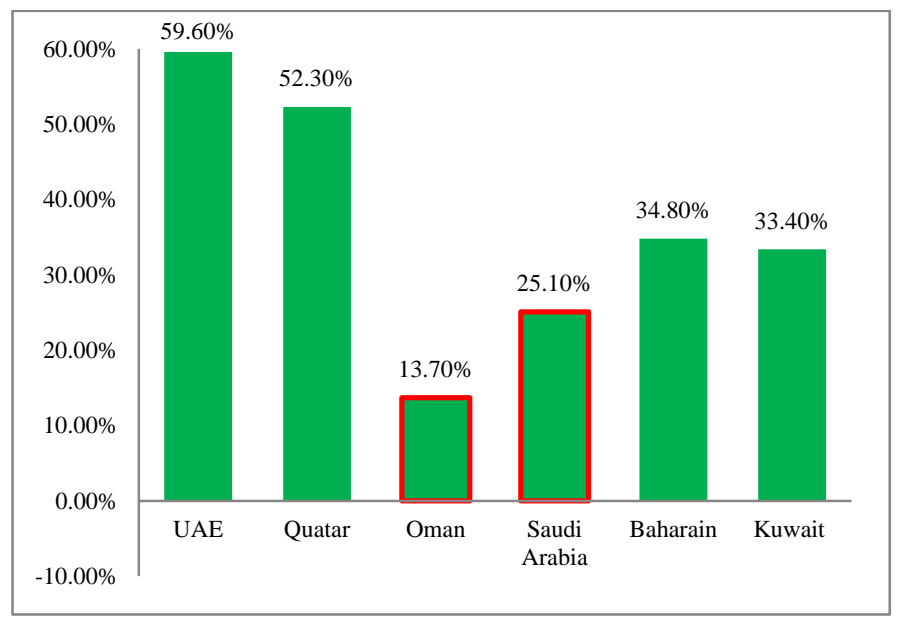

Fig. 5. Response of the students using web-based applications for learning.
It can be seen from Fig. 5 that most of the Gulf nations are penetrating towards internet as a medium of training. However, Oman is performing in a poor way as compared to the world average of $23 \%$ and Saudi Arabia (SA) also needs to enhance its percentage like in the case of UAE and Qatar. The usage of COOP training system in SA increases by implementing suitable environment for the students and parents to consider various internet based communication techniques such as emails, messaging using various social networking apps, etc.

Various reasons with respect to the companies for encouraging COOP training systems are listed in their work include:

a) improvement of the image and brand of the company,

b) reducing the burdens related with recruitment of talented and trained employees for featuring projects,

c) reducing the time and expenditure,

d) increasing the production at the reduced cost,

e) to reduce the training cost and money by training the suitable employees,

f) improving the retention rate,

$g$ ) streamlining the company employees and employer policies at the root level by training the students,

$h$ ) to ensure the professional improvement,

i) to include the social and ethnic considerations at the work place, and

j) to enhance the ability to implement the new ideas at the work place.

Various international institutions such as UNESCO (United Nations Educational Scientific and Cultural Organization) are involved in various technical and vocational training (TVT) strategies to envisage Saudi Arabia as a developing leadership in technical training. The complete training and objectives structure is designed by the technical and vocational training corporation (TVTC) and is approved by the council of Ministers Resolution No. 779 of 17 December 1969 [5]. The role of TVTC is to develop the procedures, programs and training plans for various industrial and vocational institutes (IVT). The SA government is aimed to have almost 180 industrial secondary institutes and 50 technical college colleges for both boy and girls separately so as to achieve the target of creating training and placements for $\sim 500,000$ students in the country [5]. Here the government also trying to empower girls by giving a $50-50$ chance for both boys and girls by this training programs with a plan and aim to reduce the foreign workers on its soil in various technical and vocational professions [6].

A detailed assessment report on COOP training using ABET (accreditation board for engineering and technology) was carried out by Faiz and Al-Multain. A detailed experience demonstration was given in their work using faculty course assessment report (FCAR) method for both direct and indirect assessment of course learning outcomes (CLO) [7]. A detailed comparison of student performance between Harf Al-Batin Community College (HBCC) was carried out with respect to the students of Saudi Aramco, Saudi Electricity Company 
(SEC) and Saudi Telecom Company (STC). Various influential factors were highlighted in this work as a part of strengths and weaknesses of COOP training system.

In the next section, a detailed project analysis has been carried out among the students and staff to understand the response for COOP training system in University of Hail.

\section{REQUIRED ELEMENTS OF COOP SYSTEM}

In the process of designing the web-based COOP system there are three key aspects to be realized are listed:

1) Functional and non-functional requirements

2) Usability requirements

3) Preparing the questionnaires for collecting primary data

\section{A. Functional and Non-Functional Requirements}

In a web-based COOP system, the functional requirements includes login, logout, edit, delete and search functions.

- Login Function: Used to ensure a secured gateway for the user to access the relevant information as shown in Fig. 6. Based on the participant the restrictions and access for the information can be managed by the admin. The management of University of Hail can monitor the total activity information of each participant in a secured way. Any kind of suspicious login activity will be informed to the participant and the system admin to follow further security measures to protect the personal data.

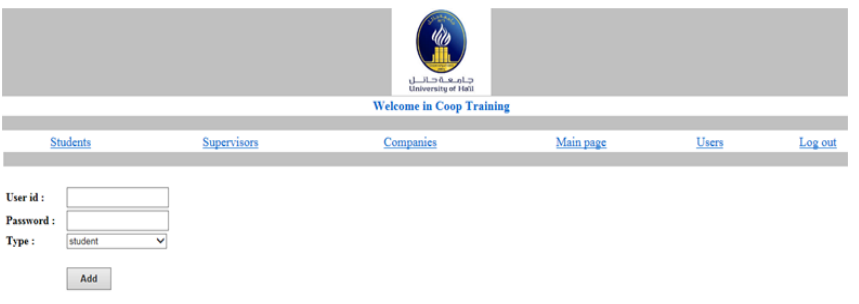

Fig. 6. Security gateway using login function

- Logout Function: This is very much essential to avoid the misusage of the information and helps to secure the privacy of the participant. The care has been taken to avoid multiple system usage and the system will logout even when the user is not active after getting into their personal account. Such provision will be useful to secure the personal data as shown in Fig. 7.

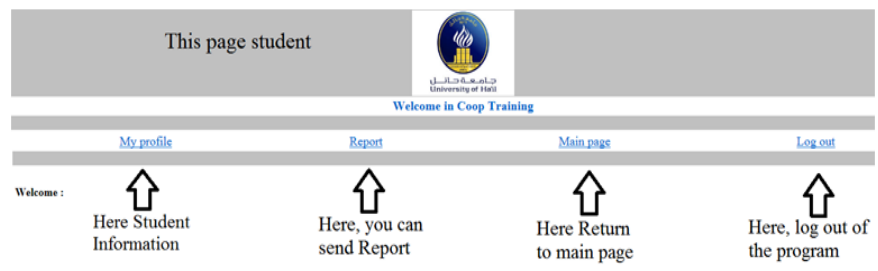

Fig. 7. Security provided using the logout function

- Add Function: Helps the participant to add the information to be shared with all other participants. For example, the supervisor will add the time-tables, project details, etc. to communicate with students and COOP training coordinator. In the similar fashion, the course coordinator can submit the assessment reports and a student can add the project reports.

- Edit Function: Helps each participant to edit the submitted information within the course time-line. This helps to update any kind of changes or event activities in an effective manner as shown in Fig. 8.

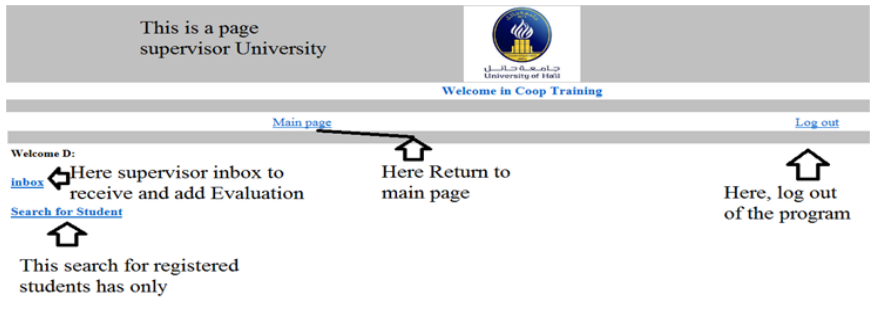

Fig. 8. Main page of the proposed COOP System

- Delete Function: Helps the participants to remove unwanted information from their account and also can avoid errors by using this function effectively.

- Search Function: It helps to find the required information from the bulk of materials in the web portal and helps in reducing the searching time for the user as shown in Fig. 9.

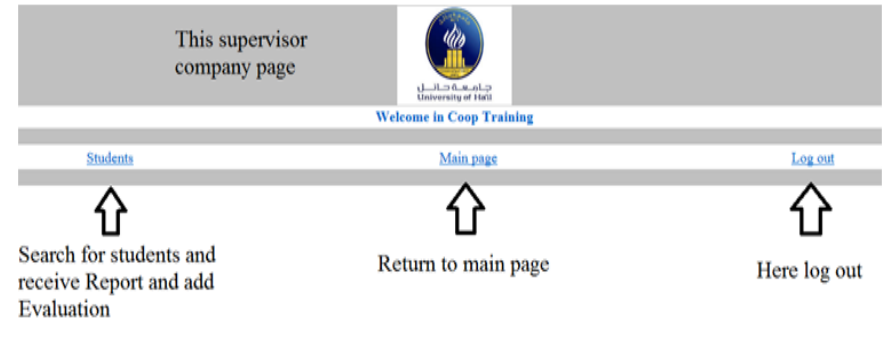

Fig. 9. Example for the Supervisor's Page

Whereas in the case of non-functional requirements it include flexibility, manageability and quality of the attributes involved in the system. These attributes are involved in controlling the authentication, security levels, usability, reliability and performance of the system.

- Flexibility: Here the flexibility does not mean that every participant can access entire information but must be able to get the defined information easily according to his status. Flexibility for accessing the information, sending and receiving the emails, uploading and downloading the information, etc.

- Manageability: Each participant must be able to manage the information according to his choice but without effecting or influencing the stipulated regulations. Students must be able to manage their search results, access the grades and reports from supervisors and trainers. Similarly, the supervisors and training coordinators must be able to manage the assessment reports and students performance summary to the seniors for necessary actions. 
- Quality: With the flexibility provided for supervisors and training coordinates will help them to understand the performance of the students with time to time updates using this web based COOP training system.

\section{B. Usability Requirements}

The usability requirements consider the easiness of system usage, matching the system with the requirements of users, effectiveness, efficiency and satisfaction towards security, performance and reliability.

\section{Preparing the Questionnaires for Collecting Primary Data}

In this work the primary data was collected by conducting interviews with the COOP trainers and supervisors to understand their experience and suggestions. In the interview carried out with the supervisors revealed that 6 sections (of which 3 male and 3 female) from software engineering, computer science and computer engineering were participated in the COOP training. Also the opinion of all students was collected at the end to assess the performance of the proposed method and its performance.

\section{FINDINGS AND ANALYSIS}

Most of the supervisors highlighted the issues related with the old system were included with paper work, communication gap between students and supervisors, requirement of additional COOP coordinators and finally the communication gap between academic supervisor and COOP trainer. One of the supervisors criticized for not having an appropriate structure to deal with the transactions and paper work always changes with supervisor to supervisor which in turn created too many issues at the time of assessment of student performance. Too much of paper work between colleges and local companies involved serious challenges to deal with and always used to miss some of the important information or lead to the confusing scenarios. The suggested specifications for the web based COOP training system needs to include a user friendly atmosphere, security for the data, safety for the personal information, trustworthy and with good quality, good performance and must be maintainable by all the participants.

On the other hand, the students participated in COOP training from University of Hail are asked to participate in a survey for which the students are provided with brief survey questionnaire and the response of the students shown in Fig. 10 towards COOP training system with the questions as well. Most of the participants in this survey expressed their satisfaction towards the COOP training system and almost all the students enjoyed the training sessions at various industries according the survey results and are willing to attend it on the regular basis. However, some students criticized this method to have an impact on their academic grades. However, most of the students were very much positive towards the training system. Apart from that the assessment method using the web based COOP system helped the students to understand the accuracy of the system assessment. However, some people are having few doubts about the success rate of the COOP training system due to lack of computer literacy in the region of Hail and expressed most of the institution they come from are not well equipped with computer and internet facilities. However, all students agreed with the accuracy, quality and effectiveness of the proposed web based COOP system to provide the best results as compared to the existing method.

Most of the students participated in the COOP training got an advantage of expressing their opinions and feedbacks.

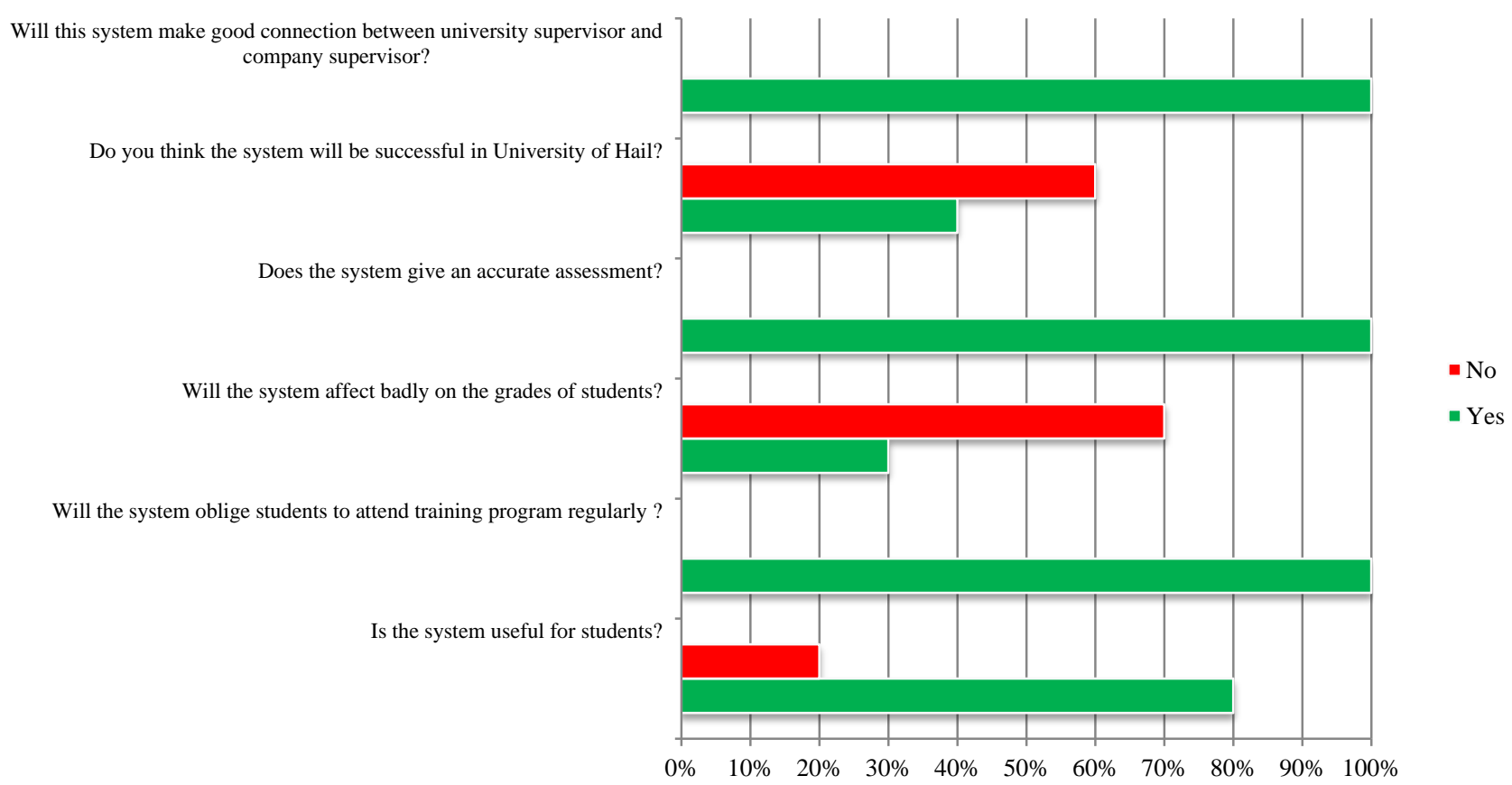

Fig. 10. Response of the students towards implementing the COOP training system. 


\section{CONCLUSIONS}

The proposed method proves to be very accurate and delivered expected results by reducing the burden of communication gap between various participants. This method allowed all the participants to interact in an effective manner by using the proposed web based COOP system for respective activities and usage. Considering the inputs of various supervisors and training coordinators the present system has been developed to help all the participants.

\section{Merits of the proposed system}

- The interaction or communication between various participants increased in an effective way.

- The companies can post their requirements and training instructions on the web portal by using the provided login account details.

- Eligible candidates list can be displayed by the college managements on the web portal for the view of both students and industries to select appropriate companies and candidates respectively.

- The paper work has been reduced and instructions are subjected to avoid overlapping of the information.

- Can consider any kind of review at any time by the management or by the participants involved within the system.

- The standard processing steps or procedures for all supervisors, training coordinators and student activities can be implemented.

- A crystal clear format for each step can be submitted and can reduce the man made errors to a greater extent.

- The supervisors and training coordinators can communicate the information of student performance or feedbacks with ease.
- In the case of indiscipline students the alerts can be raised and corrective actions can be implemented with ease.

- Project submission deadlines can be monitored by students and can submit their project reports online.

- A fair assessment of the project reports can be considered and the list of the successful candidates can be displayed on the web portal.

- Based on the previous reports, performances and feedbacks the corrective measures for the next COOP batches can be improved.

\section{ACKNOWLEDGMENT}

The authors gratefully acknowledge the support and facilities provided by the Management and Department of Computer Science and Software Engineering, University of Hail.

\section{REFERENCES}

[1] CCSIT. CO-OP Training Guidelines. KSA - Ministry of Higher Education. 2014.

[2] R. Echeng, A. Usoro and G. Majewski. "Acceptance of Web 2.0 in Learning in higher education: an empirical study of a Scottish university." In WBC July Conference Proceedings on E-learning. 2013.

[3] A. M. Aleisa and M. A. Alabdulahfez. "Cooperative education at the Riyadh College of Technology: Successes and challenges." Asia-Pacific Journal of Cooperative Education 3, no. 2 (2002): 1-7.

[4] A. S. Weber. "Web-based learning in Qatar and the GCC states." (2016).

[5] UNESCO-UNEVOC. World TVT Database - Saudi Arabia. June 2012.

[6] G. A. Khan. Technical training for half a million youth. In Arab News, April 2011.

[7] M. M. Faiz and M. S. Al-Mutairi. "Assessment of a cooperative training course using faculty course assessment report in an ABET accredited engineering technology program." In Frontiers in Education Conference (FIE), 2015 IEEE, pp. 1-7. IEEE, 2015. 